\title{
Natural history of diabetic macular streak exudates: evidence from a screening programme
}

\author{
H L Cook, R Newsom, V Long, S A Smith, J S Shilling, M R Stanford
}

\begin{abstract}
Backgroundlaims-Diabetic retinopathy screening guidelines recommend referral to an ophthalmologist if there is exudate within one disc diameter of the fovea. Many of these patients, however, have resolution of small amounts of exudate without treatment. This study aimed to assess whether patients with minimal streak or dot exudates within one disc diameter of the fovea can be monitored in a screening programme without compromising visual acuity.

Methods-A retrospective review of records and Polaroid photographs obtained by one screening centre over a 10 year period was performed. Outcomes measured were referral rates, alteration of Snellen visual acuity, and the need for macular photocoagulation treatment.

Results-55 patients (74 eyes) fulfilled entry criteria (37 streak and 37 dot exudates). Mean follow up was 56.1 months (range 12-127 months). Twenty five patients (30 eyes) were referred to an ophthalmologist. 13 eyes $(17.6 \%)$ required macular photocoagulation treatment. Four eyes (5.4\%) lost two or more lines of Snellen acuity over the follow up period (three from macular oedema and one from macular ischaemia). There was no relation between the presence or resolution of minimal exudate and visual loss ( $\mathrm{p}>0.2)$.
\end{abstract}

Ophthalmology, Rayne Institute, UMDS, St Thomas's Hospital, London

H L Cook

M R Stanford

Conclusion-It is appropriate to monitor eyes with streak or dot macular exudates at 6-9 monthly intervals in a screening programme.

(Br f Ophthalmol 1999;83:563-566)

Department of

Ophthalmology,

St Thomas's Hospital,

London

R Newsom

V Long

J S Shilling

M R Stanford

Department of

Diabetes, St Thomas's

Hospital, London

S A Smith

Correspondence to: Miss Helen L Cook, Department of

Ophthalmology, Rayne

Institute, St Thomas's

Hospital, Lambeth Palace

Road, London SE1 7EH.

Accepted for publication 25 November 1998 exudate. ${ }^{5}$ This cannot be assessed from a nonstereo screening photograph or direct ophthalmoscopy, which are the most widely used screening methods in the UK. Hence, it is not known whether eyes with small quantities of exudate require treatment or can be monitored with no threat to vision.

From clinical experience it has been noted that many cases of streak or isolated dot exudates resolve spontaneously with no adverse effect on visual acuity. Our centre has developed a protocol for closely observing patients with small quantities of macular exudate within the screening service. Patients with small amounts of streak or dot exudates are monitored by the usual screening method but at an increased frequency and with the advice of a consultant ophthalmologist. In this retrospective study we assessed whether this method of monitoring eyes with streak or dot macular exudate in a screening environment is appropriate.

\section{Patients and methods}

A retrospective review of screening records obtained by the Diabetic Eye Complication Screening (DECS) service at one centre over a 10 year period was performed. The patient population consisted of diabetic patients attending a hospital based primary eye screening clinic and were 95\% general practitioner referred and not attending hospital diabetic or ophthalmic services. At each screening visit corrected Snellen visual acuity was checked before dilatation. New visual symptoms, medical problems, or changes in diabetic treatment were recorded. A single $45^{\circ}$ Polaroid photograph of each macula was taken and direct ophthalmoscopy performed by the same experienced screener. In cases of macular exudate a consultant ophthalmologist with a special interest in diabetic retinopathy made the decision regarding appropriate rescreening interval or need to refer. This decision was based on clinical judgment and was influenced by change in visual acuity, overall progression of retinopathy, and visual function in the fellow eye. The ability of the screening service to detect true positive cases of sight threatening diabetic retinopathy (STDR) - that is, maculopathy, pre-proliferative and proliferative diabetic retinopathy, is known from a prospective study comparing the screener against the reference standard of an ophthalmologist using slit lamp biomicroscopy (unpublished data). From a sample of 302 patients the sensitivity of detection of STDR was $84 \%$ (approximate $95 \%$ CI $71 \%-97 \%)$ and the specificity was 96\% (approximate 95\% CI $89 \%-100 \%$ ). This exceeds the British Diabetic Association's guidelines for good practice in diabetic retinopathy screening. ${ }^{4}$ 


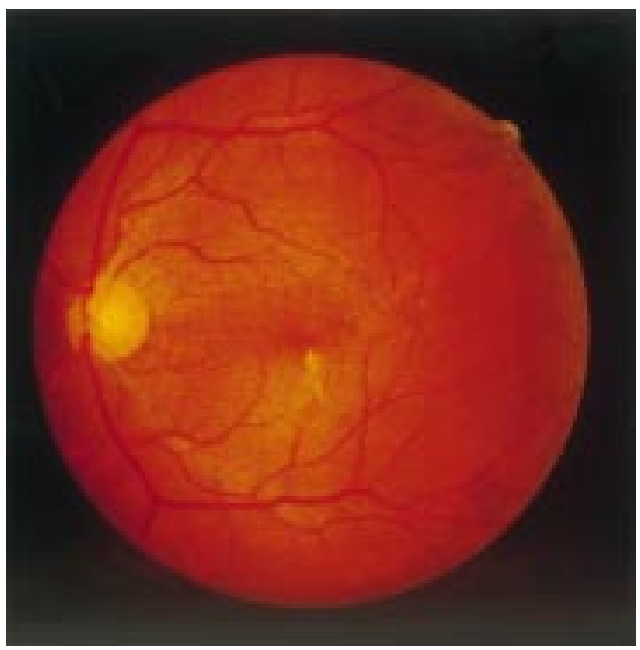

Figure 1 Fundus photograph showing streak exudate near the fovea.

Consecutive photographs and records from the screening archives were reviewed independently and assessed for the presence, nature, and location of macular exudate; corrected visual acuity; and follow up arrangements recommended by the ophthalmologist. Inclusion criteria were eyes with one to three streak (Fig 1) or one to three isolated dot (Fig 2A) exudates within one disc diameter of the fovea and a minimum follow up period of 12 months. Exclusion criteria were eyes with more than three streak or dot exudates, more exudate than Figure 3, definite circinate rings of exudate or diffusely scattered exudate, as these eyes were always referred for eye clinic review. Eyes with evidence of coexisting pre-proliferative or proliferative retinopathy were also excluded as they were automatically referred to the clinic. Hospital records of included patients referred were reviewed and findings at the ophthalmic clinic were noted, including any macular photocoagulation treatment performed. Outcomes measured were corrected visual acuity, resolution or worsening of streak or dot macular exudates, the need for referral to an ophthalmologist, and outcome of the clinic visit.

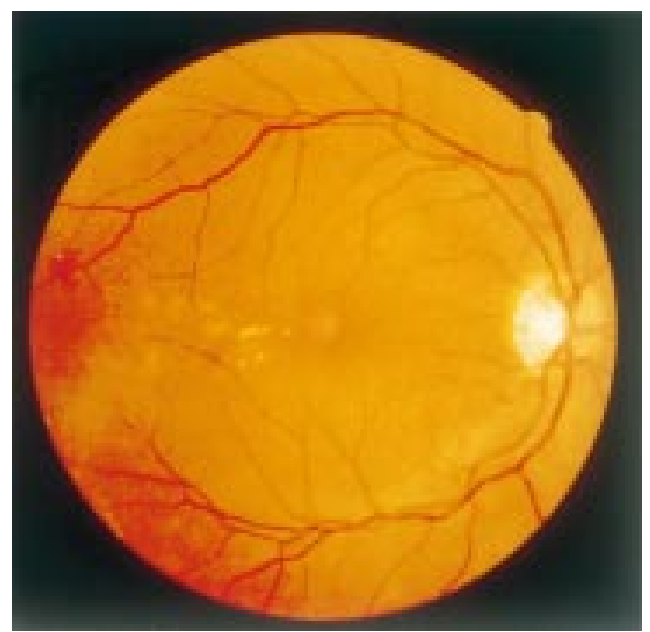

Figure 3 Eye with multiple streak exudates. All eyes included in the study had less exudate than this standard.

STATISTICAL METHODS

Statistical analysis was performed using STATVIEW (Abacus) software package. The intragroup changes were assessed using a two tailed paired $t$ test and the unpaired $t$ test was used to assess intergroup control data comparisons. $\chi^{2}$ Tests were used to measure outcomes against patient variables.

\section{Results}

Photographs and screening records from 2700 patients were reviewed, which represented all the patients seen by the DECS service at one centre over a 10 year period. Eighty three patients $(3.1 \%)$ were identified as having macular exudate within one disc diameter of the fovea, which met the entry criteria. From this sample, data from 55 patients were included in the final analysis. Twenty six patients were excluded because of inadequate follow up. This was the result of detection of exudate within the past year $(n=4)$, the patient moving out of area $(n=4)$, failure to attend outpatient or screening appointments $(n=10)$, or lost or inadequate hospital records $(n=8)$. Two patients were excluded because of concomitant serious eye disease.

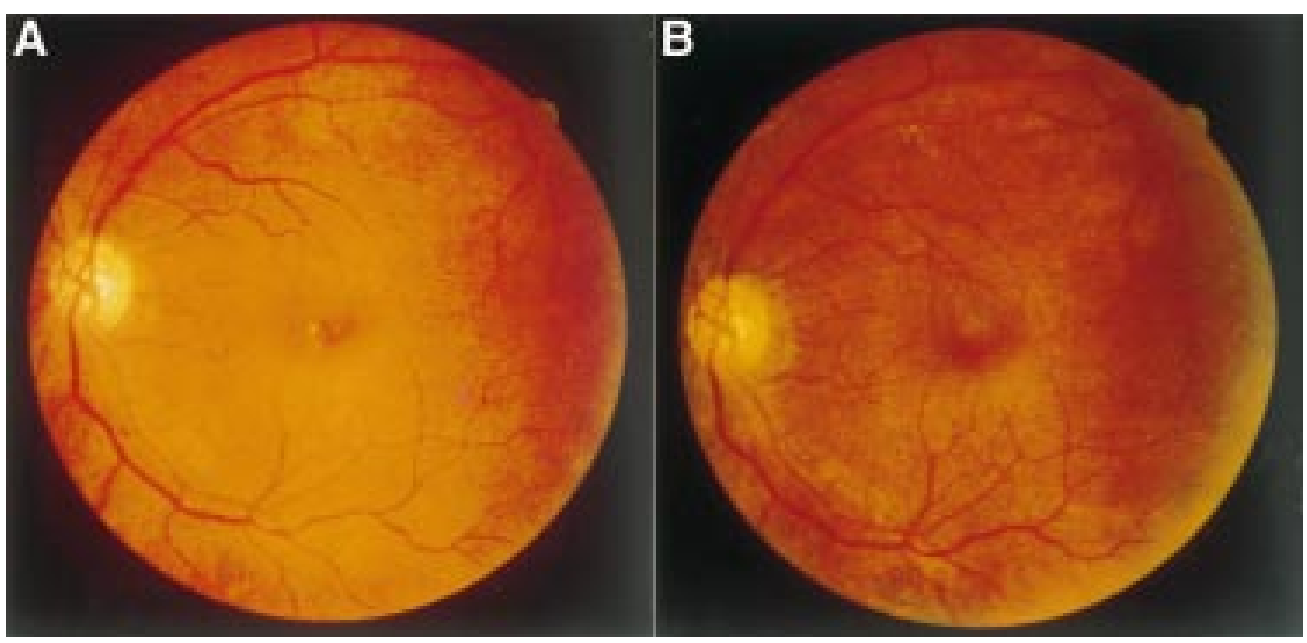

Figure 2 (A) Fundus photograph showing eye with dot exudates. (B) Same eye 6 months later when the exudate had resolved without treatment. 
Table 1 Comparison between patients excluded due to inadequate follow up and those included

\begin{tabular}{lll}
\hline & Patients included & Inadequate follow up \\
\hline Number & 55 & 28 \\
Male:female & $1: 1.6$ & $1: 1.8$ \\
Mean age (years) & $57.2($ SD 10.7) & $61.2($ SD 10.2) \\
Type of diabetes (type 1:type 2) & $1: 10$ & $1: 13$ \\
Duration of diabetes (years) & $10.76($ SD 8.5$)$ & $10.76($ SD 7.2) \\
Hypertension? & $20(36.4 \%)$ & $10(35.7 \%)$ \\
\hline
\end{tabular}

PATIENT DEMOGRAPHICS

Of the 55 diabetic patients included there were 34 women and 21 men. The mean age at detection of exudate was 57.2 years (range 25-77 years (SD 10.72)). Fifty patients had non-insulin dependent or type 2 diabetes mellitus of whom 12 were insulin treated and the remaining five patients had insulin dependent or type 1 diabetes mellitus. The mean duration of diagnosed diabetes at detection of exudate was 10.76 years (range $0.2-44$ years $(8.5)$ ). Twenty $(36 \%)$ of the patients had known hypertension. The patients excluded because of inadequate follow up were similar to this group (Table 1). The mean follow up period was 56.1 months (range 12-127 months).

\section{MACULAR EXUDATE OUTCOMES}

Of the 55 patients included in the analysis there were 74 episodes of macular exudate (37 episodes of streak and 37 of dot exudates) meeting entry criteria (37 in right and 37 in left eyes). Mean time to first review was 8.32 months (SD 5.51) with a range of $2-36$ months. Thirty nine patient episodes (52\%) were reviewed within 6 months. A further 13 episodes $(17.6 \%)$ received their first review between 6 and 9 months. All but four episodes of exudate were reviewed within 14 months. The four episodes reviewed after 14 months were due to postponement of the follow up screening appointment by the patient.

The majority of exudates resolved (Table 2). No significant relation was found between type or duration of diabetes and worsening or resolution of macular exudate. No significant relation between type of exudate, sex, age, or hypertension and resolution or increase of exudate was found ( $p>0.2$ for all).

CLINIC REFERRALS AND TREATMENT

During the follow up period 25 patients (30 eyes) were referred to the medical retina clinic: 20 patients were referred because of increasing macular exudate, however one failed to attend the clinic appointment. One was referred for unexplained worsening of visual acuity presumed to be due to maculopathy. Four were referred for other reasons; cataract precluding fundal view $(n=1)$ and progression to proliferative or pre-proliferative retinopathy $(n=3)$. Of

Table 2 Outcome of macular exudates

\begin{tabular}{lllllc}
\hline $\begin{array}{l}\text { Type of } \\
\text { exudate }\end{array}$ & Outcome & Number & $\begin{array}{l}\text { Mean time } \\
\text { (months) }\end{array}$ & $\begin{array}{l}\text { Range } \\
\text { (months) }\end{array}$ & 95\% CI \\
\hline Streak & Resolved & $25(68 \%)$ & 14.5 & $5-44$ & 3.7 \\
\multirow{2}{*}{ Dot } & Increased & $12(32 \%)$ & 16.7 & $2-56$ & 8.4 \\
& Resolved & $26(70 \%)$ & 13.4 & $5-27$ & 2.9 \\
& Increased & $8(22 \%)$ & 13 & $4-49$ & 10.4 \\
& Same & $3(8 \%)$ & 16.7 & $11-20$ & 5.58 \\
\hline
\end{tabular}

the 19 patients referred with increasing exudate, 12 patients (13 eyes) required macular focal or modified grid photocoagulation treatment for clinically significant macular oedema, as defined by the ETDRS. No eyes required full macular grid treatment. Four eyes $(31 \%)$ required treatment within 1 year of detection of exudate (mean 9.25, range 8-11 months). Three further eyes required treatment within the next 12 months. The mean period between detection of exudate and treatment was 28.54 months (range 8-83 (21.82) months). There was no significant relation between type of exudate and requirement for laser treatment $(p>0.1)$. There was no significant relation between sex, age, hypertension, type or duration of diabetes, and treatment rate ( $p>0.1$ for all).

VISUAL OUTCOME

Sixty six eyes (90\%) maintained a stable visual acuity despite fluctuation of macular exudate over the follow up period. Three eyes (4\%) gained two lines or more lines of Snellen acuity. Four eyes (5\%) lost two lines or more lines of visual acuity (95\% CI 0-11\%). Three fellow eyes with no macular exudate lost two or more lines of Snellen acuity over the same follow up period. Applying Fisher's exact test, there was no significant difference in the number of eyes with exudate losing vision compared with eyes without exudate losing vision $(p=0.69)$. One patient's vision could not be assessed at follow up owing to aphasia after a stroke.

Of the four eyes with loss of visual acuity two had increasing exudate, one developed proliferative diabetic retinopathy, and one had an ischaemic macula proved by fluorescein angiogram. The reduction of visual acuity occurred at a mean time of 23.8 months (range 9-37 months (15.34)). The loss of vision after 9 months was due to macular ischaemia. Reduction in visual acuity was not related to type of exudate, sex, age, hypertension, type or duration of diabetes $(p>0.1$ for all).

\section{Discussion}

This retrospective study attempted to assess whether it is safe to monitor small quantities of macular streak or dot exudate in a screening programme when there is a facility for review sooner than 1 year. In the population screened approximately $3 \%$ of patients developed streak or dot exudates. From the data available retrospectively there were no predictive factors to determine which patients would develop these exudates. Similarly, there were no prognostic factors to determine which patients would require photocoagulation treatment or lose visual acuity.

There are approximately 1.28 million diabetics in the UK at present. ${ }^{6}$ Therefore, from our figures, it can be extrapolated that approximately 38300 patients in the UK over a 10 year period would develop mild streak or dot macular exudates. This represents a sizeable number of clinic referrals if current screening guidelines are followed. However, in our study only four of the 74 eyes (5\%) lost two or more lines of visual acuity over a mean follow up 
period of 56.1 months although, owing to the small numbers affected, the $95 \%$ confidence intervals are relatively wide $(0-11 \%)$. One eye lost vision after 9 months as a result of macular ischaemia, which is untreatable. A further eye lost visual acuity at 12 months because of the presence of macular exudate and oedema. The other two eyes lost vision over a 37 month period. Of the 13 eyes requiring macular photocoagulation treatment, four $(31 \%)$ required treatment within 1 year of detection of exudate. The number of patients losing significant visual acuity may have been more had a standard 12 month review interval been implemented, as treatment of maculopathy would have been delayed in these patients.

Diabetic eye screening is a cost effective way of preventing loss of vision from diabetic retinopathy in both type 1 and type 2 diabetic patients. ${ }^{78}$ The natural history of proliferative retinopathy has been established. ${ }^{9}$ The markers of worsening ischaemia, such as venous beading and intraretinal microvascular abnormalities, determine urgency of referral to the ophthalmic service. ${ }^{11}{ }^{11}$ However, the natural history of maculopathy is less well understood. It is known that retinal exudates consist of plasma lipoproteins that accumulate mainly in the outer plexiform layer of the retina after leakage from altered retinal capillaries. ${ }^{12}$ It has also been noted that some exudates will spontaneously improve following thrombosis of intraretinal microaneurysms and subsequent resolution of vascular leakage. ${ }^{13}$ However, there is currently no way of predicting if or when this will occur.

Screening programmes should be as streamlined and cost efficient as possible with a high sensitivity - that is, ability to detect all the true positive cases, and a high specificity - that is, the ability to detect as few false positives as possible. Diabetic retinopathy screening guidelines advocate referral of all patients with macular exudate to an ophthalmologist for review. ${ }^{134}$ This represents a sizeable proportion of screened patients, who often have resolution of the exudate without the need for treatment and with no loss of visual acuity. Therefore, in order to streamline resources further it may be time to amend screening guidelines for maculopathy. Most screening methods in common use are unable to detect mild/moderate amounts of macula thickening and even stereopair photographs, as used in the ETDRS, are not reliable at detecting retinal thickening. ${ }^{14}$ However, most screening methods are capable of detecting macular exudates. The ETDRS criteria for significant macular exudate used a standard photograph (standard photograph no 3), ${ }^{5}$ which shows a small amount of macular exudate within one disc diameter of the fovea. Eyes in this study had dot or streak exudates, which are of a different configuration to the exudate represented in this standard. Some of the eyes had more exudate than this standard, although all had less exudate than Figure 3. The ETDRS studies did not correlate requirement and response to treatment with quantity of exudate present, as the sole criterion for treatment was the presence of clinically significant macular oedema regardless of the presence of exudate. To date there have been no data on management of patients with streak or dot exudates either with or without clinically significant macular oedema. However, from our data the number of patients at risk of requiring treatment or losing vision is small and hence a policy of monitoring is appropriate.

The screening service described is flexible owing to the close liaison with the ophthalmic department and the facility to monitor patients who would normally be referred at other centres. Our current policy is to review patients with small quantities of streak or dot macular exudate in 6-9 months and to monitor both visual acuity and fundal appearance. From this study, patients with small amounts of streak or dot exudate can be monitored in a screening programme without significant loss of visual acuity providing there is a facility for 6-9 monthly review.

This work was funded by a grant from the National Lottery Charities Fund to the Iris Fund for the Prevention of Blindness.

1 Retinopathy Working Party. A protocol for screening for diabetic retinopathy in Europe. Diabetic Med 1991;8:2637 .

2 American College of Physicians, American Diabetes Association, and American Academy of Ophthalmology. Screening guidelines for diabetic retinopathy. Ann Intern Med 1992;116:683-5.

3 Royal College of Ophthalmologists. Guidelines for diabetic retinopathy. London: RCO, 1997.

4 British Diabetic Association. Retinal photography screening for diabetic eye disease: a British Diabetic Association report. London: BDA, 1997

5 Early Treatment of Diabetic Retinopathy Study Research Group. Treatment techniques and clinical guidelines for photocoagulation of diabetic macular edema. Early treatment diabetic retinopathy study report number 2. Ophthal-

Amos AF, McCarty DJ, Zimmet P. The rising global burden of diabetes and its complications: estimates and projections to the year 2010. Diabetic Med 1997;14:S7-85.

7 Javitt JC, Aiello LP, Bassi LJ, et al. Detecting and treating retinopathy in patients with type I diabetes mellitus. Savings associated with improved implementation of current guidelines. Ophthalmology 1991;98:1565-73.

8 Javitt JC, Aiello LP, Chiang Y, et al. Preventative eye care in people with diabetes is cost-saving to the federal government. Diabetes Care 1994;17:909-17.

9 Kohner EM, McLeod D, Marshall J. Diabetic Eye Disease. In: Keen H, Jarrett J, eds. Complications of diabetes. 2nd ed. London: Arnold, 1982

10 The Diabetic Retinopathy Study Research Group. Four risk factors for severe visual loss in diabetic retinopathy. Arch Ophthalmol 1979;97:654-5.

11 Early Treatment Diabetic Retinopathy Study Research Group. Fundus photographic risk factors for progression of Group. Fundus photographic risk factors for progression of mology 1991;98(Suppl):823-33.

12 Garner A. Histopathology of diabetic retinopathy in man. Eye 1993;7:250-3

13 Hamilton AMP, Ulbig MW, Polkinghorne P. In: Hamilton AMP, Ulbig MW, Polkinghorne P, eds. Management of diabetic retinopathy. 1st ed. London: BMJ Publications, 1996: $122-9$.

14 Kinyoun J, Barton F, Fisher M, et al. Detection of diabetic macular oedema. Ophthalmoscopy versus photographyEarly Treatment of Diabetic Retinopathy Study report number 5. Ophthalmology 1989;96:746-50. 